\title{
Particle-Hole Symmetry and the Bose Glass to Superfluid Transition
}

\author{
Ranjan Mukhopadhyay and Peter B. Weichman \\ Condensed Matter Physics 114-36, California Institute of Technology, Pasadena, California 91125
}

(Received 15 November 1995)

\begin{abstract}
The generic Hamiltonian describing the zero temperature transition between the insulating Bose glass phase and the superfluid phase lacks particle-hole symmetry, but a statistical version of this symmetry is believed to be restored at the critical point. We show that the renormalization group relevance of particle-hole asymmetry may be explored in a controlled fashion only for small time dimensions, $\epsilon_{\tau} \ll 1$, where we find a stable particle-hole asymmetric and an unstable particle-hole symmetric fixed point, but we provide evidence that the two merge for some finite $\epsilon_{\tau} \approx \frac{2}{3}$, which tends to confirm symmetry restoration at the physical $\epsilon_{\tau}=1$.
\end{abstract}

PACS numbers: 74.20.Mn, 05.30.Jp, 05.70.Jk, 64.70.Pf

The zero temperature superfluid-insulator transition of bosons in a random external potential [1] is an interesting and incompletely understood example of a quantum phase transition. Experimental realizations include ${ }^{4} \mathrm{He}$ in porous media [2], granular and amorphous superconductors [3], and perhaps the pinning of flux lines by columnar defects in high temperature superconductors [4].

Through general arguments [1] and detailed Monte Carlo simulations [5], much is now understood about the basic phenomenology of this phase transition. However, important questions concerning the nature of the transition in general dimensions, $d$, remain unresolved. In particular, the detailed critical behavior in $d \geq 3$, especially the existence, or lack thereof, of an upper critical dimension $d_{c}$, is not known. Classically these issues may be addressed by expanding in $\epsilon=d_{c}-d$. However, because the disorder seen by the bosons is static, it enters the effective classical $(d+1)$-dimensional description of the problem in the form of $1 \mathrm{D}$ columns or rods. For a special case the critical behavior was analyzed by Dorogovtsev and by Boyanovsky and Cardy (DBC) [6] within a new double dimensionality expansion in $\epsilon_{\tau}$, the dimension of time, and $\epsilon=4-d-\epsilon_{\tau}$. This technique was extended to the dirty boson problem in a naive way in [7], and a mechanism for the onset of mean-field theory above $d_{c}=4$ was proposed, however, a number of technical details remained unresolved. Here we will show that a proper accounting for particle-hole symmetry not only resolves these problems, but produces an entirely new fixed point not accounted for in [7], which is proposed to be the one that actually describes the transition at the physical $\epsilon_{\tau}=1$. The mechanism for the onset of mean-field theory above $d_{c}=4$ remains unchanged.

In order to introduce the notion of particle-hole symmetry we contrast two closely related models of soft-core bosons hopping on a lattice with or without disorder. The first is the boson Hubbard model,

$$
\mathcal{H}_{B}=-\frac{1}{2} \sum_{i, j} J_{i j}\left[\hat{a}_{i}^{\dagger} \hat{a}_{j}+\hat{a}_{j}^{\dagger} \hat{a}_{i}\right]-\sum_{i} \mu_{i} \hat{n}_{i}
$$

$$
+\frac{1}{2} \sum_{i, j} V_{i j} \hat{n}_{i}\left(\hat{n}_{j}-\delta_{i j}\right)
$$

where $\hat{a}_{i}^{\dagger}$ and $\hat{a}_{i}$ are the boson creation and annihilation operators with $\hat{n}_{i}=\hat{a}_{i}^{\dagger} \hat{a}_{i}$ and commutation relations $\left[\hat{a}_{i}, \hat{a}_{j}^{\dagger}\right]=\delta_{i j}$. The second is the Josephson junction array Hamiltonian

$$
\begin{aligned}
\mathcal{H}_{J}= & -\sum_{i, j} J_{i j} \cos \left(\hat{\phi}_{i}-\hat{\phi}_{j}\right)-\sum_{i} \mu_{i} \hat{n}_{i} \\
& +\frac{1}{2} \sum_{i} V_{i j} \hat{n}_{i} \hat{n}_{j},
\end{aligned}
$$

where $\hat{\phi}_{i}$ and $\hat{n}_{i}$ are the phase and number operators at site $i$, with commution relations $\left[\hat{\phi}_{i}, \hat{n}_{j}\right]=i \delta_{i j}$. In both models $J_{i j}>0$ is the (possibly random) hopping matrix element between sites $i$ and $j, \mu_{i}$ is the random external potential at site $i$, and $V_{i j}$ is the interparticle repulsion. We will take on-site repulsion only, $V_{i j}=u_{0} \delta_{i j}$. We write $\mu_{i}=\mu-\epsilon_{i}$, where $\mu$ is the chemical potential and the disorder average $\left[\epsilon_{i}\right]_{\mathrm{av}}=0$.

The mapping between the two sets of operators is contained in the correspondence $\hat{a}_{i}^{\dagger}=\hat{n}_{i}^{1 / 2} e^{i \hat{\phi}_{i}}$ with the further constraint that we restrict the Hilbert space to states with $\hat{n}_{i} \geq 0$. A particle-hole transformation on $\mathcal{H}_{J}$ is implemented via $\hat{\phi}_{i} \rightarrow-\hat{\phi}_{i}$ and $\hat{n}_{i} \rightarrow n_{0}-$ $\hat{n}_{i}$, where $n_{0}$ is an arbitrary integer. We observe that $\mathcal{H}_{J}(\mu)=\mathcal{H}_{J}\left(\mu-n_{0} u_{0}\right)+\frac{1}{2} u_{0} n_{0}^{2}$ is, up to a constant, periodic in $\mu$ with periodicity $u_{0}=\sum_{j} V_{i j}$. It then follows that $\mathcal{H}_{J}$ is particle-hole symmetric if $\epsilon_{i} \equiv 0$ and $\mu / u_{0}$ is integer or half integer. We say that $\mathcal{H}_{J}$ has a statistical particle-hole symmetry at these same values of $\mu$ if $\epsilon_{i}$ are nonzero, but have an even probability distribution. In this case all bulk averages will be particle-hole symmetric even though the microscopic Hamiltonian is not. We will term the transition in the presence of a statistical particle-hole symmetry commensurate, all other cases being incommensurate. The 
Hamiltonian $\mathcal{H}_{B}$ can neither be periodic nor particlehole symmetric, statistically or otherwise, due to the constraint $\hat{n}_{i} \geq 0$.

These models have three possible phases [1]. For weak disorder and hopping there are incompressible Mott insulating phases with an integer $n_{0}$ particles per site that survive over a finite interval centered on $\mu=n_{0} u_{0}$. For stronger disorder there is a compressible insulating Bose glass phase in which localization effects dominate. Finally, for stronger hopping there is a superfluid phase. Of interest to us is the Bose glass to superfluid transition. It has been argued [8] that the critical point always has a statistical particle-hole symmetry, i.e., that this symmetry is restored on scales of the order of diverging correlation length. The idea is that in a disordered system close to the critical point large correlated volumes behave essentially as single coarse-grained lattice sites with large numbers of particles. As their volume diverges the distinction between adding and removing a particle disappears, and particle-hole symmetry is asymptotically restored. It is the goal of this paper to understand this phenomenon quantitatively.

We work with the effective $(d+1)$-dimensional classical action obtained from the Trotter decomposition of the partition function $Z=\operatorname{tr}\left[\exp \left(-\beta \mathcal{H}_{J}\right)\right][9,10]$ :

$$
\begin{aligned}
\mathcal{L}_{J}=\int_{0}^{\beta} d \tau\{ & -\sum_{i, j} J_{i j} \cos \left[\phi_{i}(\tau)-\phi_{j}(\tau)\right] \\
& \left.+K \sum_{i}\left[\partial_{\tau} \phi_{i}(\tau)-i \mu_{i}\right]^{2}\right\}
\end{aligned}
$$

where $K=1 / 2 u_{0}, \phi_{i}(\tau)$ is now a classical imaginary time dependent phase, and $\psi_{i}(\tau) \equiv e^{i \phi(\tau)}$ obeys periodic boundary conditions in $\tau$. The partition function is then given by the functional integral $Z=\int D \phi \exp \left(-\mathcal{L}_{J}\right)$. By relaxing the condition $\left|\psi_{i}\right|=1$, using a $|\psi|^{4}$ weighting function instead, adopting a conveniently rescaled continuum notation, and generalizing to general time dimension $\epsilon_{\tau}$, we obtain the field theoretic action

$$
\begin{aligned}
\mathcal{L}=\int d^{d} x \int d^{\epsilon_{\tau}} \tau\{ & -\frac{1}{2} \psi^{*}\left[\nabla_{\tau}-\mathbf{g}(\mathbf{x})\right]^{2} \psi+\frac{1}{2}|\nabla \psi|^{2} \\
& \left.+\frac{1}{2} r(\mathbf{x})|\psi|^{2}+u|\psi|^{4}\right\}
\end{aligned}
$$

where $\mu_{i}$ is replaced by the random $\epsilon_{\tau}$-dimensional vector $\mathbf{g}(\mathbf{x})=\mathbf{g}_{0}+\delta \mathbf{g}(\mathbf{x})$, and we take $\delta \mathbf{g}(\mathbf{x})$ to be an isotropically distributed Gaussian random variable, $\left[\delta g_{\mu}(\mathbf{x}) \delta g_{\nu}\left(\mathbf{x}^{\prime}\right)\right]_{\mathrm{av}}=\Delta_{g} \delta\left(\mathbf{x}-\mathbf{x}^{\prime}\right) \delta_{\mu \nu}$. Similarly, $r(\mathbf{x})-$ $\mathbf{g}_{0}^{2}-\delta \mathbf{g}^{2} \equiv r_{0}+\delta r(\mathbf{x})$ contains the fully particle-hole symmetric part of the disorder (corresponding to disorder in $J_{i j}$ even when $\epsilon_{i} \equiv 0$ ), and is taken to be independent of $\delta \mathbf{g}(\mathbf{x})$ with Gaussian correlations determined by $\left[\delta r(\mathbf{x}) \delta r\left(\mathbf{x}^{\prime}\right)\right]_{\mathrm{av}}=\Delta_{r} \delta\left(\mathbf{x}-\mathbf{x}^{\prime}\right)$. This differs from the coherent state formulation of (1) used in [7] in that there $\mathbf{g}(\mathbf{x}) \equiv 1$, i.e., $\Delta_{g}=0$, and the $\left|\nabla_{\tau} \psi\right|^{2}$ term is absent. We may now use the standard replica trick to average over the disorder, finally obtaining the Lagrangian $\mathcal{L}^{p}=\mathcal{L}_{1}^{p}+\mathcal{L}_{2}^{p}$, where

$$
\begin{aligned}
\mathcal{L}_{1}^{p}= & \sum_{\alpha=1}^{p} \int d^{d} x \int d^{\epsilon_{\tau}} \tau\left\{\frac{1}{2} e_{\tau}\left|\nabla_{\tau} \psi_{\alpha}\right|^{2}+\psi_{\alpha}^{*}\left(\mathbf{g}_{0} \cdot \nabla_{\tau}\right) \psi_{\alpha}+\frac{1}{2} e_{x}\left|\nabla \psi_{\alpha}\right|^{2}+\frac{1}{2} r_{0}\left|\psi_{\alpha}\right|^{2}+u\left|\psi_{\alpha}\right|^{4}\right\}, \\
\mathcal{L}_{2}^{p}= & -\frac{1}{2} \sum_{\alpha, \beta=1}^{p} \int d^{d} x \int d^{\epsilon_{\tau}} \tau \int d^{\epsilon_{\tau}} \tau^{\prime} \\
& \times\left\{\Delta_{r}\left|\psi_{\alpha}(\mathbf{x}, \vec{\tau})\right|^{2}\left|\psi_{\beta}\left(\mathbf{x}, \vec{\tau}^{\prime}\right)\right|^{2}-\Delta_{g}\left[\psi_{\alpha}^{*} \nabla_{\tau} \psi_{\alpha}-\mathbf{g}_{0}\left|\psi_{\alpha}\right|^{2}\right](\mathbf{x}, \vec{\tau}) \cdot\left[\psi_{\beta}^{*} \nabla_{\tau} \psi_{\beta}-\mathbf{g}_{0}\left|\psi_{\beta}\right|^{2}\right]\left(\mathbf{x}, \vec{\tau}^{\prime}\right)\right\},
\end{aligned}
$$

$\alpha, \beta$ being replica indices and the limit $p \rightarrow 0$ is to be taken at the end. Note that this Lagrangian does not have the periodicity of (2) or (3), retaining a commensurate transition only at $\mathbf{g}_{0}=0$, manifested now in an invariance of the action under $\vec{\tau} \rightarrow-\vec{\tau}$. Note that if $\Delta_{g}=0$ as well, the Lagrangian becomes invariant under separate time inversion for each replica, corresponding to exact particlehole symmetry which is unbroken by the disorder in $J_{i j}$. This is precisely the model studied by DBC [6]. Our aim now is to understand the relevance of $\mathbf{g}_{0}$ and $\Delta_{g}$ at the DBC fixed point.

Consider first the nature of the insulating phases for general $\epsilon_{\tau}$. We adopt a picture in which regions of the system with sufficiently large $J_{i j}$ are viewed as weakly coupled finite superfluid droplets in an insulating background, much like the grains in a granular superconductor. As the transition is approached by uniformly raising the overall magnitude of the $J_{i j}$, these droplets percolate to fill the system. A single droplet has an approximately spatially uniform phase, $\phi(\vec{\tau})$, with an effective single site "hydrodynamic" Lagrangian $[1,11]$

$$
\mathcal{L}_{\text {drop }}=\frac{1}{2} \kappa V_{\text {drop }} \int d^{\epsilon_{\tau}} \tau\left[\nabla_{\tau} \phi(\vec{\tau})-i \vec{\rho}\right]^{2},
$$

where $V_{\text {drop }}$ is the effective volume of the droplet, $\kappa$ is the compressibility, and $\vec{\rho}$ the density of the equivalent bulk superfluid if the droplet were infinitely large. Let $\hat{\mathbf{e}}_{\mu}, \mu=1, \ldots, \boldsymbol{\epsilon}_{\tau}$, be orthogonal unit vectors in $\vec{\tau}$ space. Then $\phi\left(\beta \hat{\mathbf{e}}_{\mu}\right)=\phi(\mathbf{0})+2 \pi m_{\mu}$ for some integer vector $\mathbf{m}$. The free energy may be computed exactly by performing the Gaussian integrals over the $\phi(\vec{\tau})$ and summing over all $\mathbf{m}$. The result is 


$$
f_{\text {drop }}=f_{0}-\frac{1}{\beta^{\epsilon_{\tau}}} \ln \left\{\sum_{\mathbf{l}} \exp \left[-\beta^{2-\epsilon_{\tau}}\left|\beta^{\epsilon_{\tau}-1} \kappa \vec{\rho} V_{\text {drop }}-\mathbf{l}\right|^{2} / 2 \kappa V_{\text {drop }}\right]\right\}+|\vec{\rho}|^{2} \text {, }
$$

where $\mathbf{I}$ is another integer vector and $f_{0}$ is the background free energy due to spatial fluctuations left out of (6). If $\epsilon_{\tau}=1$ then, when $\beta \rightarrow \infty$, only that $l=l_{0}$ which minimizes the exponent survives the sum. It is then easy to show that the number of particles in the droplet sticks at exactly $l_{0}(\mu)$ for the corresponding finite interval of width $1 / \kappa V_{\text {drop }}$ in $\mu$. The actual droplet density therefore has a staircase structure, but the steps become finer as the volume increases. This is a rigorous manifestation of the renormalized single site picture mentioned earlier.

If, however, $\boldsymbol{\epsilon}_{\tau}<1$ only the $\mathbf{I}=\mathbf{0}$ term survives the sum when $\beta \rightarrow \infty$, no matter how large $\vec{\rho}$ is. The actual density and compressibility therefore always vanish for a finite droplet. The Bose glass phase is thus indistinguishable from the Mott phase when $\epsilon_{\tau}<1$.

To obtain a basic understanding of where the transition to superfluidity now occurs, consider a mean-field calculation of the phase boundary. Thus we generalize (3) to general $\epsilon_{\tau}$ and take $J_{i j} \equiv J / N$ independent of $i, j$, where $N$ is the total number of sites. A single complex Hubbard-Stratanovich field, $M(\vec{\tau})$, may now be used to decouple the hopping term [1], and when $N \rightarrow \infty$ a saddle point evaluation becomes exact. The superfluid order parameter $M=\left\langle e^{i \phi}\right\rangle$ (taken real) must be determined selfconsistently from the effective single site Lagrangian,

$$
\begin{aligned}
\mathcal{L}_{\mathrm{MF}}=\int d^{\epsilon_{\tau}} \tau \sum_{i}\{ & -J M \cos \left[\phi_{i}(\vec{\tau})\right] \\
& \left.+\frac{1}{2} K\left[\nabla_{\tau} \phi_{i}(\vec{\tau})-i \vec{\mu}_{i}\right]^{2}\right\} .
\end{aligned}
$$

By perturbing in $M$ we obtain a Landau expansion for the free energy in even powers of $M$. We find a superfluid phase for $J>J_{c}(|\vec{\mu}|)$ where

$$
1 / J_{c}=\int d^{\epsilon_{\tau}} \tau \int d^{\epsilon_{\tau}} \epsilon p(\vec{\epsilon}) e^{-(1 / 2 K)|\vec{\tau}|^{2-\epsilon_{\tau}}+\vec{\tau} \cdot(\vec{\mu}-\vec{\epsilon})},
$$

where $p(\overrightarrow{\boldsymbol{\epsilon}})$ is the single site distribution for the site disorder, $\vec{\epsilon}_{i}$. For large values of $|\vec{\mu}|$ and bounded disorder, one has $J_{c} \sim \exp \left[-A|\vec{\mu}|^{q}\right]$ where $q=(2-$ $\left.\epsilon_{\tau}\right) /\left(1-\epsilon_{\tau}\right)$ and $A=\left(1-\epsilon_{\tau}\right)\left(2-\epsilon_{\tau}\right)^{-q}(2 K)^{1 /\left(1-\epsilon_{\tau}\right)}$. In Fig. 1 we plot this boundary for various $\epsilon_{\tau}$ in the absence of disorder. At the commensurate point $\mu=$ 0 one has dynamical exponent $z=1$, while on the remainder of the line one has $z=2$. In the presence of disorder the Mott lobes shrink roughly in proportion to the support of $p(\overrightarrow{\boldsymbol{\epsilon}})$, but the mean-field critical behavior is unchanged. For finite range hopping and $\epsilon_{\tau}=1 \mathrm{a}$ finite width Bose glass phase appears between the Mott and superfluid phases [1].

We now return to the double $\epsilon$ expansion in order to go beyond a mean-field description of the transition. Using (5) we carry out a momentum shell renormalization group (RG) analysis which involves first integrating out the components of the fields $\psi_{\alpha}$ with wave numbers $\mathbf{k}$ in a shell $k_{\Lambda} / b<k<k_{\Lambda}$, where $k_{\Lambda}$ is the cutoff. Next, the momenta are rescaled to restore the original cutoff $k_{\Lambda}$; frequency $\omega$ and the fields $\psi_{i}$ are also rescaled in order to keep $e_{\tau}=e_{x} \equiv 1$. In the limit $b \rightarrow 1$ we generate RG flows, which to lowest nontrivial order for the commensurate case, $\vec{\mu}=0$, are

$$
\begin{aligned}
\dot{\bar{r}} & =2 \bar{r}+\frac{2(m+1) \bar{u}}{1+\bar{r}}-\frac{2 \bar{\Delta}_{r}}{1+\bar{r}}+O\left(\bar{u}^{2}, \bar{\Delta}_{r}^{2}, \bar{\Delta}_{g}^{2}\right), \\
\dot{\bar{u}} & =\epsilon \bar{u}-2(m+4) \bar{u}^{2}+12 \bar{u} \bar{\Delta}_{r}+O\left(\bar{u}^{3}, \ldots\right), \\
\dot{\bar{\Delta}}_{r} & =\left(\epsilon+\epsilon_{\tau}\right) \bar{\Delta}_{r}+8 \bar{\Delta}_{g}^{2}-4(m+1) \bar{u} \bar{\Delta}_{r}+O\left(\bar{u}^{3}, \ldots\right), \\
\dot{\bar{\Delta}}_{g} & =\bar{\Delta}_{g}\left(\epsilon+\epsilon_{\tau}+10 \bar{\Delta}_{r}-2\right)-2 \bar{\Delta}_{g}^{2}+O\left(\bar{u}^{3}, \ldots\right),
\end{aligned}
$$

where $\quad \bar{r}=r / k_{\Lambda}^{2}, \quad \bar{u}=K_{d} u, \quad \bar{\Delta}_{r}=K_{d} \Delta_{r}, \quad$ and $\bar{\Delta}_{g}=k_{\Lambda}^{2} K_{d} \Delta_{g}, \quad K_{d}=2 /(4 \pi)^{d / 2} \Gamma(d / 2), \quad$ and $m=1$ is the number of boson species. For small $\epsilon_{\tau}$ the only fixed point occurs at $\bar{\Delta}_{g}=0$, and one obtains the usual DBC random rod fixed point [6], i.e., exact particlehole symmetry is restored at criticality. However, for $\epsilon_{\tau}>\epsilon_{\tau}^{c} \equiv[8(2 m-1)-3(m+2) \epsilon] /(13 m+16)$ this fixed point becomes unstable, and a new one with $\bar{\Delta}_{g}^{*}=[(13 m+16) / 8(2 m-1)]\left(\epsilon_{\tau}-\epsilon_{\tau}^{c}\right) \quad$ is found. For $m=1$ and $\epsilon=0$ (corresponding to $d=3$ at $\epsilon_{\tau}=1$ ), one obtains $\epsilon_{\tau}^{c}=8 / 29$. This is an uncontrolled

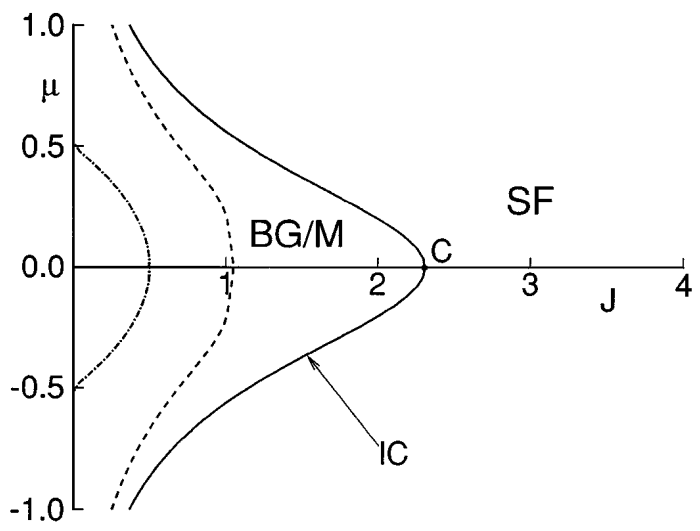

FIG. 1. The mean-field phase diagram for the nondisordered Josephson junction model, showing Bose glass/Mott (BG/M) and superfluid (SF) phases, with incommensurate line (IC) and commensurate point (C) for $\epsilon_{\tau}=0.5,0.9,1.0$, with larger $\epsilon_{\tau}$ corresponding to a smaller Mott lobe. Note that only one Mott lobe persists for $\epsilon_{\tau}<1$. For $\epsilon_{\tau}=1$ the Mott lobe shown is actually repeated periodically, with one centered on each integer value of $\mu$. 
estimate, as $\epsilon_{\tau}^{c}$ does not vanish with $\epsilon$, but its small size is encouraging. We see then that "anomalous" rescaling due to higher order diagrams actually makes $\Delta_{g}$ more relevant than $\Delta_{r}$, and there appears a new dirty boson fixed point, corresponding to a nontrivial commensurate transition. To lowest order, $\bar{r}^{*}, \bar{u}^{*}, \Delta_{r}^{*}$, and the exponent $\nu$ remain unchanged from their DBC values, but the dynamical exponent $z=1+\bar{\Delta}_{r}^{*}+\bar{\Delta}_{g}^{*}=$ $\left[(m+4) \epsilon+(7 m+10) \epsilon_{\tau}\right] / 4(2 m-1)$ is substantially larger than before, consistent with the expectation $z=d$ at $\epsilon_{\tau}=1$ [1].

Finally, let us include the $\mathbf{g}_{0}$ term. To linear order the flow equation for $g_{0}=\left|\mathbf{g}_{0}\right|$ is found to be $\dot{g}_{0}=g_{0}[1+$ $\left.\bar{\Delta}_{r}-\bar{\Delta}_{g}\right]$. This term becomes irrelevant at the dirty boson fixed point for $\epsilon_{\tau} \geq \epsilon_{\tau 1}=(4 / 3)(2 m-1) /(1+$ $m-\epsilon)$. For $m=1$ and $\epsilon=0$ one obtains $\epsilon_{\tau 1}=2 / 3$. This is again an uncontrolled estimate, but does indeed indicate that the statistical symmetry is restored prior to $\epsilon_{\tau}=1$. For $\epsilon_{\tau}<\epsilon_{\tau 1}$ there is a new incommensurate fixed point at nonzero $g_{0}$. In order to locate it one should choose $z$ to keep $g_{0}$, rather than $e_{\tau}$, fixed during the RG flow. The flow equation for $\Delta_{g}$ becomes

$$
\dot{\bar{\Delta}}_{g}=\bar{\Delta}_{g}\left[\epsilon+\epsilon_{\tau}-4+8 \bar{\Delta}_{r}\right]+8 \bar{\Delta}_{r}^{2},
$$

while the remaining ones are identical to those in [7]. The fixed point found in that work did not account for $\Delta_{g}$, but we see that if $\Delta_{r}^{*}=O\left(\epsilon, \epsilon_{\tau}\right)$ then $\Delta_{g}^{*}=O\left(\epsilon^{2}, \epsilon \epsilon_{\tau}, \epsilon_{\tau}^{2}\right)$, so the results given there are indeed correct to $O\left(\epsilon, \epsilon_{\tau}\right)$. However, as $\epsilon_{\tau}$ grows, so does $\Delta_{g}^{*}$, eventually leading to the merging with the dirty boson fixed point.

We also note that the $\left|\nabla_{\tau} \psi_{\alpha}\right|^{2}$ term which was ignored in [7] has the flow equation $\dot{e}_{\tau}=-\left(1+\bar{\Delta}_{r}\right) e_{\tau}+\bar{\Delta}_{g}$, implying that it is of the same order as $\bar{\Delta}_{g}^{*}$ at the fixed point. A nonzero $e_{\tau}$ fixes the convergence problems found in [7].

Finally, to see how the two fixed points merge we write down flow equations in the intermediate region, $\epsilon_{\tau 1}>\epsilon_{\tau}>\epsilon_{\tau}^{c}$, where one must consider both $e_{\tau}$ and $\bar{g}_{0} \equiv k_{\Lambda} g_{0}$. We now choose $z$ so that $e_{\tau}+\bar{g}_{0} \equiv 1$. We find then that $\dot{g}_{0}=\left(2+2 \bar{\Delta}_{r}-z\right) g_{0}$ with $z=(1+$ $\left.\bar{\Delta}_{r}+\bar{\Delta}_{g}\right)+\left(1+\bar{\Delta}_{r}\right) \bar{g}_{0}$. At the fixed point we therefore find $\bar{g}_{0}^{*}=\left(1+\bar{\Delta}_{r}^{*}-\bar{\Delta}_{g}^{*}\right) /\left(1+\bar{\Delta}_{r}^{*}\right)$, which vanishes precisely when $g_{0}$ becomes irrelevant at the dirty boson fixed point.

To summarize, for small $\epsilon_{\tau}$ the unstable DBC fixed point and stable incommensurate fixed point exist. For $\epsilon_{\tau}^{c}<\epsilon_{\tau}<\epsilon_{\tau 1}$ there are three fixed points, with the new commensurate dirty boson fixed point being more stable than the DBC fixed point, but less stable than the incommensurate fixed point. Finally, for $\epsilon_{\tau}>\epsilon_{\tau 1}$ the incommensurate fixed point merges with the dirty boson fixed point, which is then completely stable. This provides a detailed scenario by which statistical particlehole symmetry is restored (see Fig. 2). We caution,

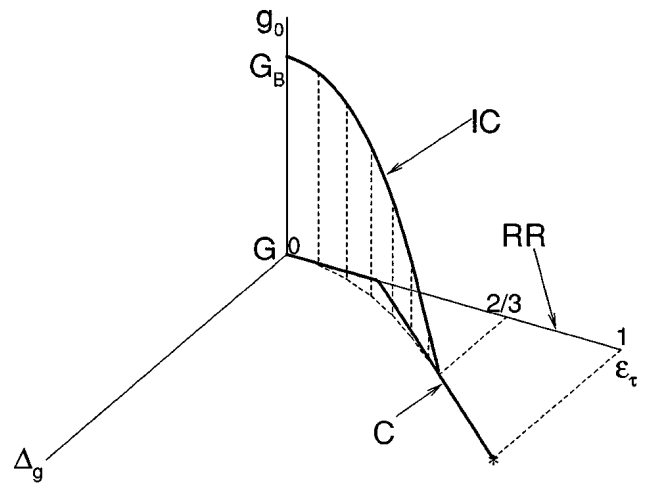

FIG. 2. Proposed behavior of the random rod (RR), incommensurate (IC), and commensurate dirty boson (C) fixed points as functions of $\epsilon_{\tau}$. Here $G$ and $G_{B}$ are commensurate and incommensurate Gaussian fixed points. We propose that only $\mathrm{C}$ is stable at $\epsilon_{\tau}=1$, and that it describes the physical dirty boson problem.

however, that, due to both the uncontrolled nature of the double $\epsilon$ expansion at the dirty boson fixed point and the absence of separate Bose glass and Mott phases for $\epsilon_{\tau}<1$, extrapolation of any of these results to $\epsilon_{\tau}=1$ is highly suspect. The general scenario we propose, however, seems very natural and illuminating.

This research was supported by the Sloan Foundation and the NSF under Grant No. DMR-9308205.

[1] M.P. A. Fisher, P. B. Weichman, G. Grinstein, and D. S. Fisher, Phys. Rev. B 40, 546 (1989).

[2] See P. A. Crowell, F. W. Van Keuls, and J.D. Reppy, Phys. Rev. Lett. 75, 1106 (1995), and references therein.

[3] See K. Kim and P. B. Weichman, Phys. Rev. B 43, 13583 (1991); E. S. Sørensen, M. Wallin, S. M. Girvin, and A.P. Young, Phys. B 49, 12115 (1994), and references therein.

[4] D. R. Nelson and V. M. Vinokur, Phys. Rev. B 48, 13060 (1993).

[5] R. T. Scalettar, G. G. Batrouni, and G. T. Zimanyi, Phys. Rev. Lett. 66, 3144 (1991); M. Makivic, N. Trivedi, and S. Ullah, Phys. Rev. Lett. 71, 2307 (1993); P. B. Weichman, Phys. Rev. Lett. 74, 1038 (1995); S. Zhang, N. Kawashima, J. Carlson, and J.E. Gubernatis, Phys. Rev. Lett. 74, 1500 (1995).

[6] S. N. Dorogovtsev, Phys. Lett. 76A, 169 (1980); D. Boyanovsky and J. L. Cardy, Phys. Rev. B 26, 154 (1982).

[7] P. B. Weichman and K. Kim, Phys. Rev. B 40, 813 (1989).

[8] M. P. A. Fisher, Physica (Amsterdam) 177A, 553 (1991); A. T. Dorsey and M. P. A. Fisher, Phys. Rev. Lett. 68, 694 (1992).

[9] P. B. Weichman and R. Mukhopadhyay (to be published).

[10] Y. Tu and P. B. Weichman, Phys. Rev. Lett. 73, 6 (1994).

[11] M.E. Fisher and V. Privman, Phys. Rev. B 32, 447 (1985). 